\title{
Experimental demonstration of deep traps in silica-based polyethylene nanocomposites by combined isothermal surface potential decay and pulsed electro- acoustic measurements
}

Y. Wang, M. Hao, Z. Xu, D. Qiang, G. Chen, and A. Vaughan

Citation: Appl. Phys. Lett. 113, 022904 (2018); doi: 10.1063/1.5025633

View online: https://doi.org/10.1063/1.5025633

View Table of Contents: http://aip.scitation.org/toc/apl/113/2

Published by the American Institute of Physics

\section{Articles you may be interested in}

Switching Purcell effect with nonlinear epsilon-near-zero media

Applied Physics Letters 113, 021103 (2018); 10.1063/1.5030023

Optical bistability in shape-memory nanowire metamaterial array

Applied Physics Letters 113, 021105 (2018); 10.1063/1.5025400

Enhanced stability of perovskite solar cells using hydrophobic organic fluoropolymer

Applied Physics Letters 113, 023902 (2018); 10.1063/1.5038917

Nonlinear frequency conversion in one dimensional lithium niobate photonic crystal nanocavities

Applied Physics Letters 113, 021104 (2018); 10.1063/1.5039948

Graphdiyne under pressure: A Raman study

Applied Physics Letters 113, 021901 (2018); 10.1063/1.5023619

Mid-wavelength high operating temperature barrier infrared detector and focal plane array

Applied Physics Letters 113, 021101 (2018); 10.1063/1.5033338

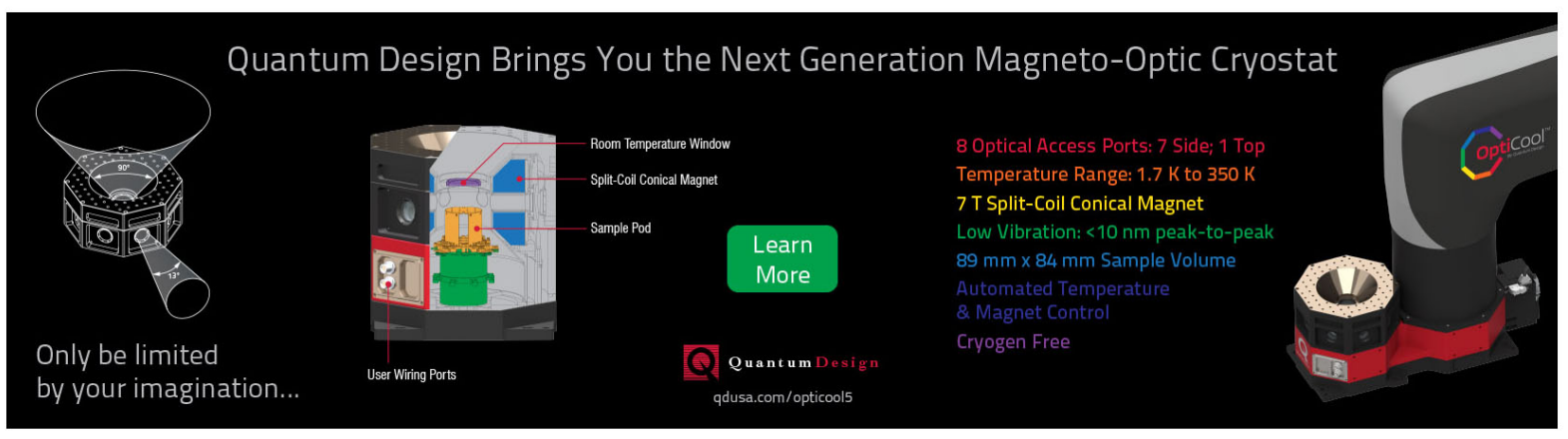




\title{
Experimental demonstration of deep traps in silica-based polyethylene nanocomposites by combined isothermal surface potential decay and pulsed electro-acoustic measurements
}

\author{
Y. Wang, ${ }^{\text {a) }}$ M. Hao, Z. Xu, D. Qiang, G. Chen, and A. Vaughan \\ The Tony Davis High Voltage Laboratory, University Road, SO17 1BJ Southampton, United Kingdom
}

(Received 11 February 2018; accepted 14 June 2018; published online 13 July 2018)

\begin{abstract}
The ability to suppress space charge accumulation at high electric fields makes nanocomposites attract significant research interest as potential insulation materials in high-voltage direct current cable development. At present, the deep trap introduced by nanoparticles is frequently applied to be responsible for the observed space charge suppression in nanocomposites. However, the experimental results that support deep-trap formation have not been rigorously examined. We therefore propose herein a simple and more direct approach based on isothermal surface potential decay combined with pulsed electro-acoustic measurements to verify the presence of deep traps in silicabased blend polyethylene nanocomposites. The results indicate that the deep traps are indeed introduced by filling nanosilica and the space charge suppression observed in the nanocomposite with a low loading ratio is caused by deeply trapped charges in the sub-surface region of specimens. Published by AIP Publishing. https://doi.org/10.1063/1.5025633
\end{abstract}

The benefits of nanotechnology have spread to various areas, such as aerospace and medicine. Although the research on applying nanocomposites in the dielectric realm started relatively late, the number of publications dealing with this topic is growing rapidly. Many experimental results have shown the improved electrical properties after filling nanoparticles into conventional polymeric insulation materials such as polyethylene and epoxy. ${ }^{1}$ One of the major improvements offered by nanocomposites is the ability to suppress the accumulation of space charge when stressing at high electric fields. ${ }^{2}$ This feature is particularly useful for high-voltage direct current (HVDC) applications, especially the power cable, for instance, where the accumulation of space charge in dielectric materials has been the major obstacle to the reliable operation of the HVDC transmission systems.

Charge transport mechanisms have been widely applied to describe and understand the electrical properties of nanocomposites. Factors such as the electric field, which influence the space charge behavior, can strongly impair the electrical performance of dielectric materials. In contrast to nanocomposites, the charge transport of polymeric dielectrics has been studied for decades, and several numerical models have been established to describe space charge dynamics within these materials. ${ }^{3}$ Upon filling a polymeric dielectric with nanoparticles, the changed electrical performance of these nanocomposites is often attributed to the effect of nanoparticles on charge transport. For specifically realizing the mechanisms of this, several models have been proposed. ${ }^{1}$ However, apart from emphasizing the importance of the interphase between nanoparticles and the polymer matrix, the mechanisms of charge transport in nanocomposites are still perplexed according to these models.

Takada et $a .^{2}$ claimed that the formation of the deep traps due to filling nanoparticles is the key to revealing the mechanism of charge transport in nanocomposites. Hereafter, the deep

\footnotetext{
a) Author to whom correspondence should be addressed: yw14g13@soton.ac.uk.
}

traps have been implemented to explain the electrical properties observed in nanocomposites such as conduction current. ${ }^{4}$ Some experimental studies verified the presence of deep traps in nanocomposites based on thermally stimulated current (TSC) and pulsed electro-acoustic (PEA) methods. ${ }^{5,6}$ However, these supporting results are not convincing and leave room for doubt. Regarding the TSC technique, the results can be affected not only by the magnitude of the applied electric field during the polarization stage but also by the bias voltage owing to charge recombination during the measurement stage. Moreover, the TSC results do not provide a spatial distribution of traps in the specimen. Conversely, the PEA is able to provide a spatial distribution of space charge across the specimen, but its spatial resolution is limited (typically $5-10 \mu \mathrm{m}$ ). ${ }^{7}$ Accordingly, this technique cannot distinguish space charges located in the subsurface region of the specimen from capacitive charges on the electrodes, as illustrated in Fig. 1. No significant difference can be observed between Figs. 1(a) and 1(b), but space charge becomes evident when it departs away from the electrodes, as shown in Fig. 1(c). Therefore, two scenarios are possible when no bulk charge is detected in the specimen: (i) no space charge exists in the specimen and (ii) the space charge is situated close to electrodes. Therefore, considering the drawbacks of measuring techniques including both TSC and PEA, the existence of deep traps in nanocomposites is worth further confirming by different approaches. Additionally, there is a widely held view that space charge suppression is only observed in the nanocomposites with loading ratios usually less than 1 weight percent (wt. $\%$ ) since other publications have reported the obviously accumulated space charge with complicated dynamics in the bulk of nanocomposites with a high loading ratio. ${ }^{1,2,8,9}$ These mentioned experimental results further confuse the understanding about the effect of deep traps on the mechanism of space charge suppression, especially stemming from a lack of consideration in nanoparticle loading ratios.

Herein, a simple experimental procedure based on the combination of isothermal surface potential decay (ISPD) ${ }^{7,10}$ 

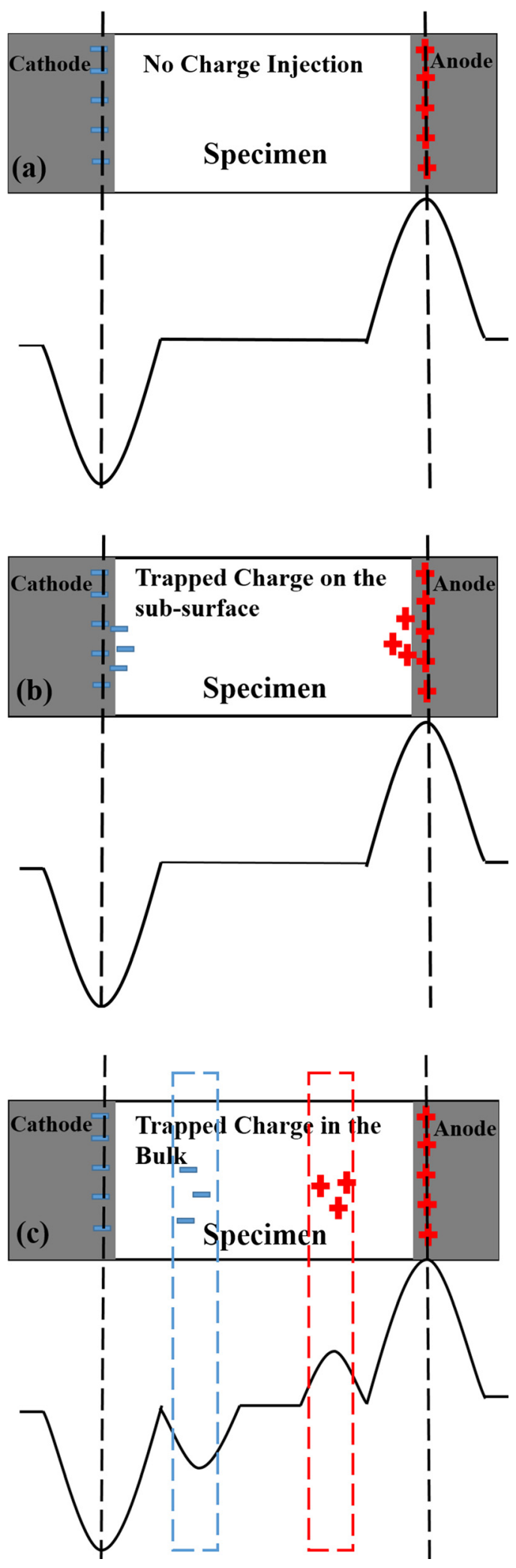

FIG. 1. Schematic outputs from a PEA system with limited spatial resolution with (a) no charge injection, (b) injected charges trapped close to electrodes, and (c) charge trapped in the bulk.

and PEA measurements is proposed to verify the presence of the deep traps in nanocomposites. Additionally, an explanation of the mechanism related to the effect of deep traps on space charge suppression is offered.

In order for experiments to be conducted explicitly, the following hypotheses are proposed: (1) nanoparticles can induce deep traps within composites, (2) and correspondingly, the suppression of space charge in nanocomposites with a low loading ratio is achieved by the deep traps in the sub-surface region of a specimen as shown in Fig. 1(b), which can capture charges injected from the electrodes. These captured charges produce an electric field that opposes the applied electric field, thereby reducing the electric field at the interface between electrodes and the specimen. Since the charge injection strongly depends on the electric field, further charge injection from the electrodes is suppressed. This suppression of charge injection continues unless the trapped charges move further into the bulk of specimen.

Based on the above hypotheses, charges could be deeply trapped in the sub-surface region of specimens when stressing under a high DC electric field. Although these charges would not be detected by the PEA technique due to limited spatial resolution, they can be experimentally detected by using other techniques. In accordance with the location of these deeply trapped charges, the surface potential decay measurement is therefore adopted. If the obtained results can show the deeply trapped charges in the sub-surface of nanocomposites with a low loading ratio in contrast to the unfilled specimen, the proposed hypothesis will be confirmed. In addition, the space charge results of the specimens measured in this research will be provided.

To test these hypotheses, the material used was silica based blend polyethylene nanocomposites. The initial idea of developing the $20 \%$ high-density polyethylene (Rigidex HD5813A BP Chemical) blend with $80 \%$ low-density polyethylene (LD100BW ExxonMobil Chemicals) was to offer a recycle thermoplastic insulation material to substitute crosslinked polyethylene used in the HVDC cable, and the properties of this blend polyethylene have been tested and reported exhaustively since the idea was proposed. ${ }^{9,11}$ Afterward, many species of nanoparticles were filled into the blend polyethylene, especially nanosilica, which has shown homogeneous dispersion and consistency in the electrical properties including dielectric response, scanning electron microscopy, breakdown strength, etc. from batch to batch specimen manufacturing. ${ }^{8,9,12,13}$ In addition, nanosilica is a potential particle for industrial applications because it can be produced by flame hydrolysis or polymerization at low cost and is widely available in a range of nanometers from tens to hundreds. In this research, as-received nanosilica (Sigma-Aldrich 637238) was used. The size of this nanosilica is around $10 \mathrm{~nm}-20 \mathrm{~nm}$, and no additional surface treatment was functionalized. Three different specimens, including unfilled blend polyethylene referred to as "BPE," a low loading ratio nanocomposite with $0.5 \mathrm{wt}$. \% nanosilica referred to as " $0.5 \mathrm{wt}$. $\%$," and a high loading ratio nanocomposite with 5 wt. \% nanosilica referred to as " $5 \mathrm{wt}$. \%" were tested. The fabrication of these specimens followed previous work. ${ }^{8,9}$ The thickness of specimens was $120 \pm 5 \mu \mathrm{m}$, and the area of specimens was $20 \pm 5 \mathrm{~cm}^{2}$.

The process of combined measurements was divided into two procedures. Step one: the specimens were first stressed in the PEA measurement system for $2 \mathrm{~h}$ at $50 \mathrm{kV} /$ $\mathrm{mm}$ and at ambient temperature. The applied electric field is sufficient to ensure charge-carrier injection because it is much greater than $8 \mathrm{kV} / \mathrm{mm}$, which is the reported threshold 


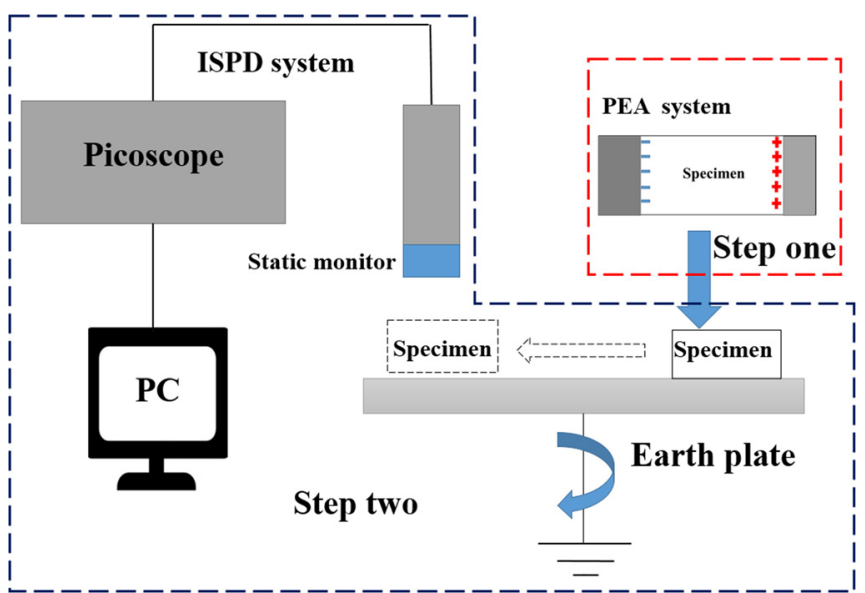

FIG. 2. Schematic configuration of combined PEA and ISPD.

field for low-density polyethylene. ${ }^{14}$ The space charge profiles of the specimens were recorded during the stressing period. Step two: after $2 \mathrm{~h}$, the specimens were quickly transferred from the PEA system to the ISPD system with minimized possible charge dissipation during the transfer, and the specimen surface near the anode in the PEA system faces the static monitor in the ISPD system. The surface potential decay of the stressed specimens was measured during the next $1500 \mathrm{~s}$. The schematic configuration of this combined PEA and ISPD system is illustrated in Fig. 2. The semiconducting polymer is placed on the top cylindrical electrode of the PEA system, and an aluminum plate is used as the bottom electrode. More details about PEA and ISPD systems were described in the previous work. ${ }^{7,9}$ To ensure repeatability, the experiments were repeated three times for each type of specimen.

As shown in Fig. 3, the space charge results of BPE indicate that positive charges quickly accumulate in the bulk of the specimen near the cathode (from $0 \mu \mathrm{m}$ to about $50 \mu \mathrm{m}$ ) within the first $2 \mathrm{~min}$ of applying the voltage. The space charge remains relatively stable for the next $2 \mathrm{~h}$. Upon stressing at $50 \mathrm{kV} / \mathrm{mm}$, little space charge is detected within the bulk of 0.5 wt. $\%$ as shown in Fig. 4 . In other words, for the $2 \mathrm{~h}$ period during which the voltage is applied, filling $0.5 \mathrm{wt}$. \% nanosilica

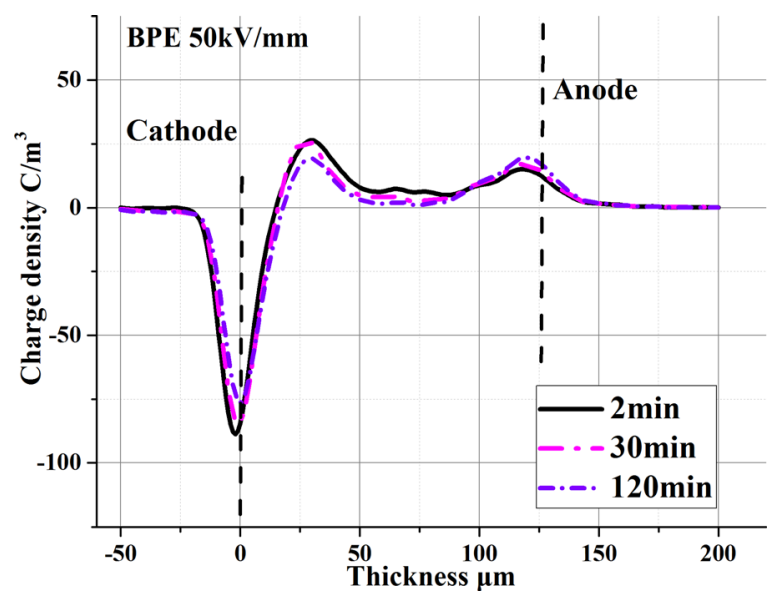

FIG. 3. Space charge profile of blended polyethylene under an applied electric field of $50 \mathrm{kV} / \mathrm{mm}$.

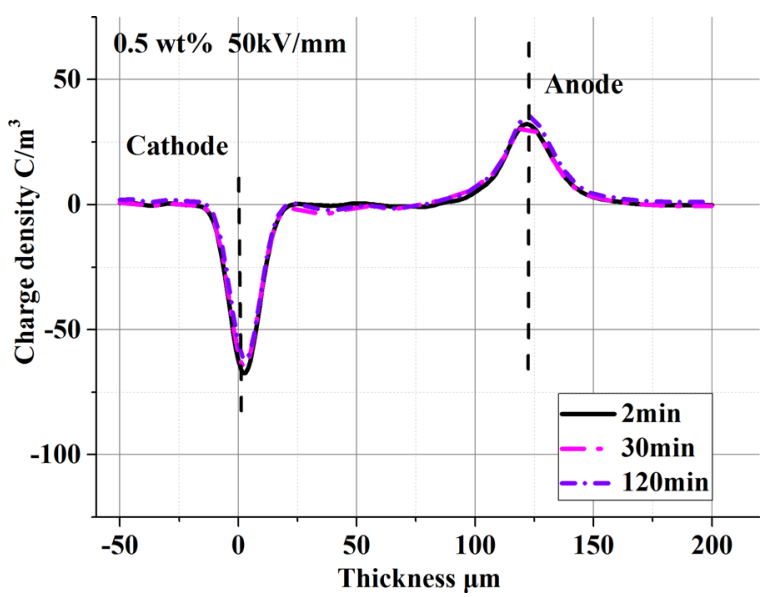

FIG. 4. Space charge profile of nanodielectrics with a loading concentration of $0.5 \mathrm{wt}$. \% under an applied electric field of $50 \mathrm{kV} / \mathrm{mm}$.

can effectively suppress space charge accumulation in contrast to BPE, which is consistent with that reported in the literature. However, as illustrated in Fig. 4, the space charge near the electrodes is barely distinguishable due to the limited spatial resolution of the PEA system. Hence, the information extracted according to these obtained results is not enough to verify which scenario either Fig. 1(a) or Fig. 1(b) should be adopted to explain the mechanism of space charge suppression.

Regarding $5 \mathrm{wt} . \%$, the space charge profiles (see Fig. 5) indicate that a large amount of heterocharges, which is more than BPE, accumulates in the bulk of the specimen. These results are consistent with the previously reported results, ${ }^{8,9}$ which show that space charge suppression is lost for high nanosilica loading ratios. The space charge results shown above clearly demonstrate the influence of the nanosilica loading ratio on the space charge characteristics. However, confirming the hypotheses based solely on the results of PEA measurements is insufficient. To obtain more information on the presence of deep traps and their impact on charge transport, the ISPD results of stressed specimens are presented.

After stressing for $2 \mathrm{~h}$ at $50 \mathrm{kV} / \mathrm{mm}$ in the PEA system, the stressed specimens were quickly transferred to the ISPD measurement system to measure the decay of the surface potential. During the PEA stressing, part of injected charges

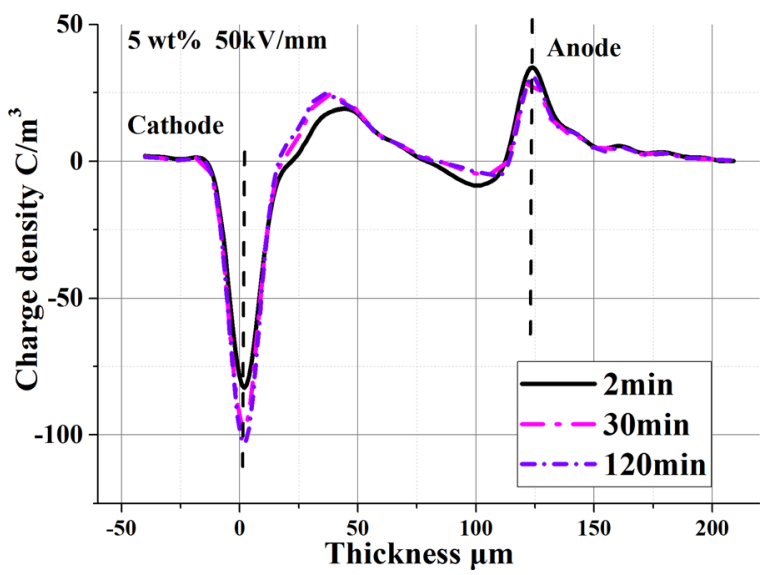

FIG. 5. Space charge profile of nanodielectrics with a loading concentration of $5 \mathrm{wt}$. \% under an applied electric field of $50 \mathrm{kV} / \mathrm{mm}$. 
can transport through the bulk toward to the opposite electrode while the charges captured in the sub-surface determine the initial surface potential. Usually, the high potential and the slow decay curve reflect the presence of deeply trapped charges in the material. ${ }^{10}$ Figure 6 shows the ISPD results of the stressed specimens. The largest surface potential $(0.51 \mathrm{kV})$ occurs for $0.5 \mathrm{wt}$. \%, and the surface potential decay rate of this specimen is the slowest of the three specimens (i.e., it takes about $1400 \mathrm{~s}$ to reach the steady state). Compared with the surface potential decay of the BPE, which merely starts from $0.45 \mathrm{kV}$ and gradually decreases to the steady state over a period of $1000 \mathrm{~s}$, the presence of deeply trapped charges in $0.5 \mathrm{wt}$. \% is clearly verified. Thus, the results shown in Fig. 4 verify that the space charge profile of $0.5 \mathrm{wt} . \%$ is in line with the situation shown in Fig. 1(b). The charges are deeply trapped in the sub-surface of $0.5 \mathrm{wt}$. \%. According to the importance of the electric field in charge injection and the observed slow surface potential decay, the space charge suppression in $0.5 \mathrm{wt}$. \% stems from the reduced electric field at the interface, which is due to the opposite electric field generated by these deeply trapped charges in the sub-surface region. Based on the above results and analysis, the hypotheses proposed are confirmed.

On the other hand, the initial surface potential of $5 \mathrm{wt}$. $\%$ is much less than that of $0.5 \mathrm{wt} . \%$ and BPE. The surface potential decay of $5 \mathrm{wt}$. \% starts from about $0.25 \mathrm{kV}$ and quickly reaches the steady state (at about $0.24 \mathrm{kV}$ ) within 200 s. Considering the heterocharges revealed in Fig. 5, the charges that should be trapped in the sub-surface region are quickly transported through the bulk to the other electrode during PEA stressing. Only a few charges remain trapped in the sub-surface region, resulting in the low surface potential seen in Fig. 6. As a result, the decay of 5 wt. \% is stable because this low potential is insufficient to cause further transport of these trapped charges through the bulk. Compared with 0.5 wt. $\%, 5$ wt. \% should induce more deep traps in the sub-surface, and thus, more obvious space charge suppression should be observed. However, the opposite results presented in Figs. 5 and 6 indicate that the deeply

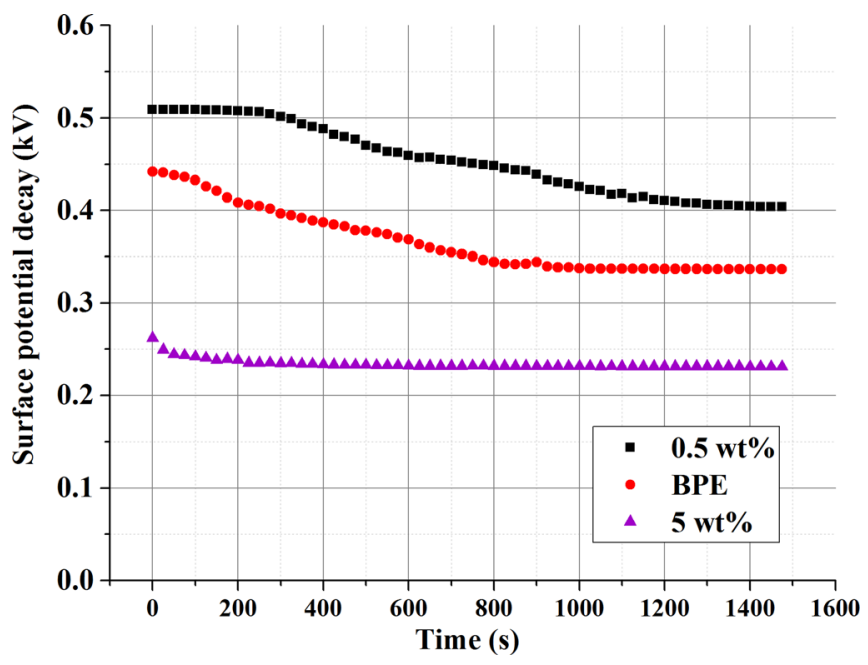

FIG. 6. Decay of surface potential for the three specimens after stressing in the PEA system. trapped charges in the sub-surface of $5 \mathrm{wt}$. \% continue to move further into the bulk of the specimen. A reasonable explanation of this phenomenon was proposed by the charge transport model based on the tunnelling process. ${ }^{4,15}$ When stressing at high electric fields, the trapped charge carriers quickly and easily tunnel through deep traps because the distance between adjacent traps in nanocomposites with high loading ratios is sufficiently short to make the tunnelling effect dominate charge transport. Thus, more deep traps located in the bulk become involved in the charge transport as the loading ratio of nanoparticles increases, and the recombination between positive and negative charges is easy to occur during this process. In this work, the deeply trapped charges in the sub-surface region of $5 \mathrm{wt}$. \% can be transported easily and faster through the bulk via the adjacent deep traps located in the bulk, whereas this is difficult to occur in $0.5 \mathrm{wt}$. \% due to the large distance between these deep traps. After the deeply trapped charges moved to the bulk, the electric field at the interface will not be reduced, which cause more charges to be injected and transported through 5 wt. \%. In addition, the heterocharges near the anode in 5 wt. $\%$ as illustrated in Fig. 5 indicate that the negative charge can recombine with the positive charge trapped in the sub-surface region of the specimen. Thus, the amount of trapped positive charge is reduced, which further lowers the initial surface potential of specimen $5 \mathrm{wt}$. \% as shown in Fig. 6.

This work experimentally verifies the deep-trap mechanism for the nanocomposites by combining a series of PEA and ISPD measurements. This experimental approach overcomes the drawbacks of the conventional TSC method and the PEA method. The PEA results show the suppressed space charge accumulation in the nanocomposite with a low nanosilica loading ratio $(0.5 \mathrm{wt}$. \%). By comparing the results of the unfilled blend polyethylene and the nanocomposite, the conclusions are drawn as follows: (1) deep traps are introduced in the composites upon filling nanosilica and (2) charges deeply trapped in the sub-surface region of nanocomposites loaded with 0.5 wt. \% nanosilica are the main reason, causing the observed space charge suppression.

${ }^{1}$ T. Tanaka and T. Imai, IEEE Electr. Insul. Mag. 29(1), 10 (2013).

${ }^{2}$ T. Takada, Y. Hayase, Y. Tanaka, and T. Okamoto, IEEE Trans. Dielectr. Electr. Insul. 15(1), 152-160 (2008).

${ }^{3}$ J. Zhao, Z. Xu, G. Chen, and P. L. Lewin, J. Appl. Phys. 108(12), 124107 (2010).

${ }^{4}$ G. Li, G. Chen, and S. Li, Appl. Phys. Lett. 109(6), 062901 (2016).

${ }^{5}$ B. Han, X. Wang, Z. Sun, J. Yang, and Q. Lei, Appl. Phys. Lett. 102(1), 012902 (2013).

${ }^{6}$ X. Huang, P. Jiang, and Y. Yin, Appl. Phys. Lett. 95(24), 242905 (2009).

${ }^{7}$ Z. Xu, L. Zhang, and G. Chen, J. Phys. D: Appl. Phys. 40(22), 7085 (2007).

${ }^{8}$ K. Lau, A. Vaughan, G. Chen, I. Hosier, A. Holt, and K. Y. Ching, IEEE Trans. Dielectr. Electr. Insul. 21(1), 340 (2014).

${ }^{9}$ Y. Wang, G. Chen, and A. Vaughan, "Space charge dynamics in silicabased polyethylene nanocomposites," in 2014 IEEE Conference on Electrical Insulation and Dielectric Phenomena (CEIDP) (2014), pp. 727-730.

${ }^{10}$ W.-W. Shen, H.-B. Mu, G.-J. Zhang, J.-B. Deng, and D.-M. Tu, J. Appl. Phys. 113(8), 083706 (2013). 
${ }^{11}$ I. Hosier, A. Vaughan, and S. Swingler, J. Mater. Sci. 32(17), 4523 (1997).

${ }^{12}$ I. Hosier, M. Praeger, A. Vaughan, and S. Swingler, IEEE Trans. Dielectr. Electr. Insul. 24(5), 3073 (2017).

${ }^{13}$ K. Lau, A. Vaughan, G. Chen, I. Hosier, and A. Holt, J. Phys. D: Appl. Phys. 46(9), 095303 (2013)
${ }^{14}$ N. Liu, C. Zhou, G. Chen, and L. Zhong, Appl. Phys. Lett. 106(19), 192901 (2015).

${ }^{15}$ G. Chen, S. Li, and L. Zhong, "Space charge in nanodielectrics and its impact on electrical performance," in 2015 IEEE 11th International Conference on the Properties and Applications of Dielectric Materials (ICPADM) (2015), pp. 36-39. 